\title{
Generalizing Source Geometry of Site Contamination by Simulating and Analyzing Analytical Solution of Three-Dimensional Solute Transport Model
}

\author{
Xingwei Wang, ${ }^{1}$ Jiajun Chen, ${ }^{1}$ Hao Wang, ${ }^{2}$ and Jianfei Liu ${ }^{3}$ \\ ${ }^{1}$ Key Laboratory for Water and Sediment Sciences of Ministry of Education, School of Environment, Beijing Normal University, \\ Beijing 100875, China \\ ${ }^{2}$ CECEP L\&T Environmental Technology Co. Ltd., Beijing 100085, China \\ ${ }^{3}$ School of Civil Engineering, Henan Polytechnic University, Jiaozuo 454003, China
}

Correspondence should be addressed to Jiajun Chen; jeffchen@bnu.edu.cn

Received 28 February 2014; Accepted 13 May 2014; Published 13 August 2014

Academic Editor: Oluwole Daniel Makinde

Copyright (C) 2014 Xingwei Wang et al. This is an open access article distributed under the Creative Commons Attribution License, which permits unrestricted use, distribution, and reproduction in any medium, provided the original work is properly cited.

\begin{abstract}
Due to the uneven distribution of pollutions and blur edge of pollutant area, there will exist uncertainty of source term shape in advective-diffusion equation model of contaminant transport. How to generalize those irregular source terms and deal with those uncertainties is very critical but rarely studied in previous research. In this study, the fate and transport of contaminant from rectangular and elliptic source geometry were simulated based on a three-dimensional analytical solute transport model, and the source geometry generalization guideline was developed by comparing the migration of contaminant. The result indicated that the variation of source area size had no effect on pollution plume migration when the plume migrated as far as five times of source side length. The migration of pollution plume became slower with the increase of aquifer thickness. The contaminant concentration was decreasing with scale factor rising, and the differences among various scale factors became smaller with the distance to field increasing.
\end{abstract}

\section{Introduction}

Concern about contamination of the subsurface environment has greatly stimulated research of solute transport phenomena in porous media. A number of solute transport studies aimed at solving the advective-diffusion equation (ADE) for nonreactive and reactive solutes, subject to various initial and boundary conditions [1]. Several analytical solutions for one-, two-, and three-dimensional ADEs have been developed for predicting the transport of various contaminants in the subsurface [2]. For instance, Ogata and Banks [3], Sauty [4], and Van Genuchten [5] formulated several analytical solutions for the one-dimensional ADE subject to the first-type (Dirichlet), second-type (Neumann), and third-type (Cauchy) boundary conditions, respectively. Batu $[6,7]$ compiled analytical solutions to two-dimensional ADE with various source boundary conditions. The analytical solutions for three-dimensional ADE have been derived by Sagar [8], Domenico [9], Leij et al., [10], Batu [11], Sim and
Chrysikopoulos [12], and Park and Zhan [13]. Analytical solutions for ADE play important roles in giving initial or approximate estimates of contaminant distributions in soil or aquifer systems [14]. Although large amounts of two- or three-dimensional analytical models are available at present [15], those models illustrated the surface condition for transport from a regular source.

The regular sources included point source, line source, and area source (rectangular or elliptic). For example, Sim and Chrysikopoulos [12] investigated the effect of aquifer boundary conditions and the source geometry on solute transport in subsurface porous formations. Porous media with either semi-infinite or finite thickness and source geometry with either a point source or an elliptic source were examined. Park and Zhan [13] tested the sensitivity of the line source solutions to source geometry, dispersion coefficients, and distance to the source. The results indicated that the concentration at a near field point was sensitive to the source geometry when the dispersion coefficients are anisotropic, 
and it was less sensitive to the source geometry when the dispersion coefficients were isotropic. The concentration at a far field was found to be almost independent of the source geometry. Zhao et al. [16] investigated the effects of different domain shapes in general and trapezoidal domain shape in particular on the morphological evolution of NAPL dissolution fronts in two-dimensional fluid-saturated porous media. Domain shapes had a significant effect on the propagating speed, and an increase in the divergent angle of a trapezoidal domain could lead to a decrease in the propagating speed of the NAPL dissolution front. The above-mentioned studies commendably analyzed the fate and transport of contaminant under various conditions from a regular source geometry. However, due to the complex condition in reality, such as uneven distribution of pollutions and blur edge of pollutant area, the shape of pollution plume is not explicit in the initial period of contaminated site investigation. On this background, there will exist uncertainty of source term shape in $\mathrm{ADE}$ model of contaminant transport. How to generalize those irregular source terms and deal with those uncertainties is very critical and can influence the accuracy of final calculation result. Nevertheless, few previous studies have been conducted concerning those problems and then provided decision-support for site remediation practice, through numerical simulation methods.

Therefore, the objective of this study is to develop a guideline for source geometry generalization, which differs from previous research in two aspects. First, it can answer the questions of "how to use the regular source geometry to substitute the irregular source geometry" and "what regular source geometry can be used for substitution." Second, it can help technicians examine and predict the contaminant transport in subsurface flow systems in actual site remediation. We start from identifying contaminant from regular (i.e., rectangular and elliptic) source geometry, investigating its application scope and condition from its analytical solutions of ADE as well as the corresponding influence factors. Then, simulating flow and transport of contaminants from two regular source geometries under the same condition compare flow and transport of contaminant based on the simulation results to develop source geometry generalization guideline. The obtained results will provide useful information and technical support for estimating the distribution of contaminant concentration in the initial period of contaminated site investigation.

\section{Mathematical Model}

This study considers the problem of contaminant transport in saturated, homogeneous porous media, accounting for threedimensional hydrodynamic dispersion in a uniform flow field, and first-order decay of liquid phase and sorbed contaminants with different decay rates. Before formulating the model, several assumptions must be made. First, it is assumed that the upper and lower boundaries of homogeneous porous media are impermeable (no-flow boundary); if a water table boundary exists, the slope of the water table is small and the aquifer is horizontal without curvature and parallel to the lower boundary. Second, there exists one-dimensional

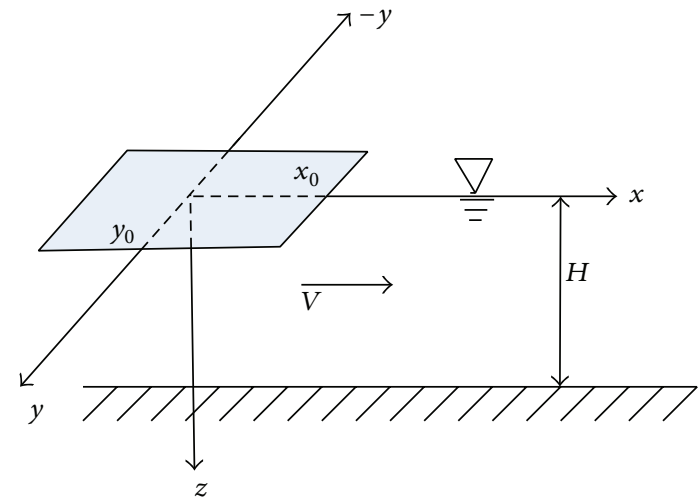

FIGURE 1: The general geometry of the rectangular source.

steady-state ground water flow along the $x$-axis, and the averaged actual flow velocity is $u$. Third, cardinal direction of dispersion coefficient is consistent with the coordinate axis. Fourth, there is no pollution in the study area at initial moments, and the continuous injection strength is $I_{a}$.

Based on the above assumptions, the advective-diffusion equation can be formulated by the following partial differential equation:

$$
\frac{\partial C}{\partial t}=D_{x} \frac{\partial^{2} C}{\partial x^{2}}+D_{y} \frac{\partial^{2} C}{\partial y^{2}}+D_{z} \frac{\partial^{2} C}{\partial z^{2}}-u \frac{\partial C}{\partial x}-\lambda C+\frac{I_{a}}{n} .
$$

The corresponding initial and boundary conditions are as follows:

$$
\begin{gathered}
\left.C\right|_{t=0}=0, \quad-\infty<x, \quad y<\infty, \quad 0<z<H, \\
\left.C\right|_{x \rightarrow \pm \infty}=0, \quad-\infty<y<\infty, \quad 0<z<H, \quad t>0, \\
\left.C\right|_{y \rightarrow \pm \infty}=0, \quad-\infty<x<\infty, \quad 0<z<H, \quad t>0, \\
\left.\frac{\partial C}{\partial z}\right|_{z=z_{\text {table }}}=\left.\frac{\partial C}{\partial z}\right|_{z=H}=0, \quad-\infty<x, \quad y<\infty,
\end{gathered}
$$

$$
t>0
$$

where $C$ denotes the solute concentration; $D_{x}, D_{y}$, and $D_{z}$ are the longitudinal, lateral, and vertical hydrodynamic dispersion coefficients, respectively; $u$ represents the averaged actual steady-state pore water velocity; $t$ is time; $x, y$, and $z$ stands for the spatial coordinates in the longitudinal, lateral, and vertical directions, respectively; $\lambda$ is the decay rate of solutes; $n$ is the porosity of the porous medium; $I_{a}$ is the continuous injection strength; $H$ denotes aquifer thickness.

2.1. Analytical Solution of Rectangular Source. The general geometry of the rectangular source is show in Figure 1. The origin of the coordinate system is at the upper boundary. The positive $z$-axis is downward. The aquifer is assumed infinite in the $x$-and $y$-directions but finite in the $z$-direction with a thickness of $H$. The rectangular source of contamination is located on top of an aquifer, without considering its thickness influence, with $x \in\left[0, x_{0}\right], y \in\left[-y_{0}, y_{0}\right]$, 


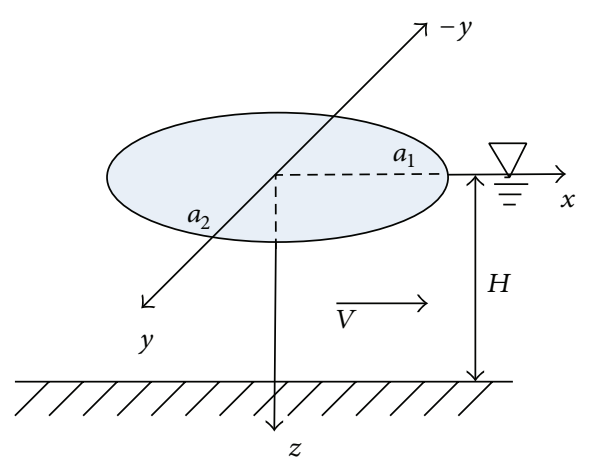

FIGURE 2: The general geometry of the elliptic source.

and $z \in[0, H]$, which is described mathematically by the following expression:

$$
I_{a}= \begin{cases}I_{0} f(t), & -x_{0}<x<x_{0},-y_{0}<y<y_{0}, \quad z_{\text {table }}<z<H \\ 0, & \text { otherwise. }\end{cases}
$$

Through using Green's function methods, three-dimensional analytical solution for rectangular source can be obtained [13] as follows:

$$
\begin{aligned}
& C(x, y, z, t) \\
& =\frac{1}{4 n H} \\
& \times \int_{0}^{t} I_{a}(t-\tau) \exp (-\lambda t) \\
& \times\left[\operatorname{erfc} \frac{x-u \tau-x_{0}}{2 \sqrt{D_{x} \tau}}-\operatorname{erfc} \frac{x-u \tau+x_{0}}{2 \sqrt{D_{x} \tau}}\right] \\
& \times\left[\operatorname{erfc} \frac{y-y_{0}}{\left.2 \sqrt{D_{y} \tau}-\operatorname{erfc} \frac{y+y_{0}}{2 \sqrt{D_{y} \tau}}\right]}\right. \\
& \times\left[1+2 \sum_{n=1}^{\infty} \cos \frac{n \pi\left(z-z_{\text {table }}\right)}{H} \exp \left(-\frac{D_{z} n^{2} \pi^{2}}{H^{2}} \tau\right)\right] d \tau,
\end{aligned}
$$

where $I_{0}$ is a constant and $f(t)$ is a function of time; $\operatorname{erfc}[x]$ denotes residual error function, equal to $1-(2 / \sqrt{\pi}) \int_{0}^{x} e^{-z^{2}} d z$.

2.2. Analytical Solution of Elliptic Source. Consider solute movement from an elliptic source as sketched in Figure 2. The set of coordinate system is similar to the rectangular source, the source contaminant is located on top of an aquifer, and the solute may move from the source, which has a negligible thickness, by diffusion or advection, with $x \in\left[0, a_{1}\right], y \in$ $\left[-a_{2}, a_{2}\right]$, and $z \in[0, H]$; the elliptic source geometry is defined mathematically by the following expression:

$$
I_{a}= \begin{cases}I_{0} f(t), & \frac{x^{2}}{a_{1}^{2}}+\frac{y^{2}}{a_{2}^{2}} \leq 1 \\ 0, & \text { otherwise. }\end{cases}
$$

The governing solute transport equations are solved analytically by employing Laplace, Fourier, and finite Fourier cosine transform techniques:

$$
\begin{aligned}
& C(x, y, z, t) \\
& \left.\begin{array}{c}
\frac{1}{4 n H \sqrt{\pi D_{x}}} \\
\times \int_{0}^{t} \int_{-a_{1}}^{a_{1}} I_{a}(t-\tau) F_{1}(\tau) \\
\times\left\{\int_{0}^{\tau} F_{2}(\tau) F_{3}(x-q, p) F_{4}(p) F_{5}(p) \frac{d p}{\sqrt{p}}\right. \\
\left.+\frac{F_{3}(x-q, \tau)}{\sqrt{\tau}} F_{4}(\tau) F_{5}(\tau)\right\} d p d \tau, \\
F_{1}(t)=\exp \left[-\left(r_{2}+\lambda_{5}\right) t\right], \\
F_{2}(t)=I_{1}\left(2 \sqrt{r_{1} r_{2} p(t-p)}\right) \sqrt{\frac{r_{1} r_{2} p}{t-p},} \\
=\exp \left[\frac{u x}{2 D_{x}}-\frac{x^{2}}{4 D_{x} t}-t\left(r_{1}+\lambda-r_{2}-\lambda_{5}+\frac{u^{2}}{4 D_{x}}\right)\right], \\
F_{4}(t)=\operatorname{erfc}\left[k_{2}(q, y, t)\right]-\operatorname{erfc}\left[k_{1}(q, y, t)\right], \\
k_{1}(q, y, t)=\frac{y+Y}{2 \sqrt{D_{y} t}} ; \quad k_{2}(q, y, t)=\frac{y-Y}{2 \sqrt{D_{y} t}} ; \\
F_{5}(t)=1+2 \sum_{n=1}^{\infty} \cos \frac{n \pi\left(z-z_{\text {table }}\right)}{H} \exp \left(-\frac{D_{z} n^{2} \pi^{2}}{H^{2}} t\right), \\
a_{1}^{2}
\end{array}\right]
\end{aligned}
$$

where $r_{1}$ and $r_{2}$ are the forward and reverse rate coefficients; $\lambda$ is decay rate of liquid phase solute; $\lambda_{5}$ is decay rate of sorbed contaminant.

\section{Model Simulations and Discussion}

Model simulations are performed for two different source configurations. The integrals present in the analytical solutions are evaluated numerically by using globally adaptive quadrature algorithms. The number of terms is selected so that additional terms do not alter the summation more than 
TABLE 1: Model simulation parameters for two source geometries.

\begin{tabular}{|c|c|c|c|}
\hline \multicolumn{2}{|c|}{ Elliptic source geometry } & \multicolumn{2}{|c|}{ Rectangular source geometry } \\
\hline Parameter & Value & Parameter & Value \\
\hline$\lambda$ and $\lambda \mathrm{s}$ & 0.01 day $^{-1}$ & $\lambda$ and $\lambda s$ & 0.01 day $^{-1}$ \\
\hline$u$ & $5 \mathrm{~cm} \mathrm{day}^{-1}$ & $u$ & $5 \mathrm{~cm} \mathrm{day}^{-1}$ \\
\hline$I_{a}$ & $100 \mathrm{mg} \mathrm{cm}^{-2}$ day $^{-1}$ & $I_{a}$ & $100 \mathrm{mg} \mathrm{cm}^{-2}$ day $^{-1}$ \\
\hline$D_{x}$ & $2000 \mathrm{~cm}^{2}$ day $^{-1}$ & $D_{x}$ & $2000 \mathrm{~cm}^{2}$ day $^{-1}$ \\
\hline$D_{y}$ & $500 \mathrm{~cm}^{2}$ day $^{-1}$ & $D_{y}$ & $500 \mathrm{~cm}^{2}$ day $^{-1}$ \\
\hline$D_{z}$ & $500 \mathrm{~cm}^{2}$ day $^{-1}$ & $D_{z}$ & $500 \mathrm{~cm}^{2}$ day $^{-1}$ \\
\hline$r_{1}$ & 0.02 day $^{-1}$ & $Z_{\text {table }}$ & $500 \mathrm{~cm}$ \\
\hline$r_{2}$ & 0.01 day $^{-1}$ & $H$ & $200 \mathrm{~cm}$ \\
\hline$Z_{\text {table }}$ & $500 \mathrm{~cm}$ & $x_{0}$ & $50 \mathrm{~cm}$ \\
\hline$H$ & $200 \mathrm{~cm}$ & $y_{0}$ & $25 \mathrm{~cm}$ \\
\hline$a_{1}$ & $100 /(\pi)^{0.5} \mathrm{~cm}$ & & \\
\hline$a_{2}$ & $50 /(\pi)^{0.5} \mathrm{~cm}$ & & \\
\hline
\end{tabular}

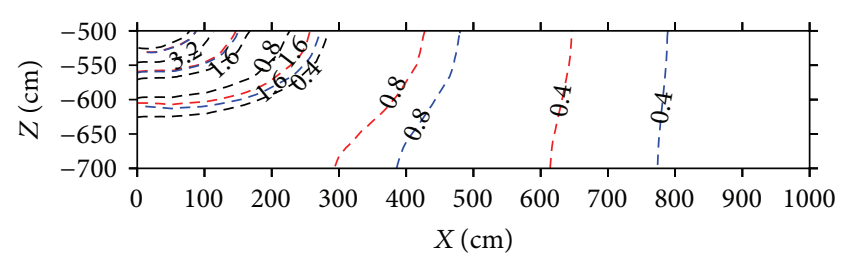

FIgURE 3: Contaminant concentration contours on the $x z$-plane $(y=0)$ at $t=10, t=100$, and $t=500$ days under conditions for rectangular source geometry.

$1.0 E-7$. In order to compare the fate and transport of contaminant from rectangular and elliptic source geometry, it is assumed that aspect ratio (length-width ratio for rectangular source and major axis-minor axis ratio for elliptic source) of rectangular source geometry is the same as that of elliptic source geometry; besides that, the area of two disparate sources is equal, which can guarantee the uniform total pollution load under the same continuous injection strength condition.

3.1. Rectangular Source Type. The groundwater table and the bottom of the finite thickness aquifer are assumed to be located at $z=z_{\text {table }}=0 \mathrm{~cm}$ and $z=H=200 \mathrm{~cm}$, respectively. Other parameters are shown in Table 1; with them, concentrations can be calculated at any given time for continuous source with consideration of the first-order decay using FORTRAN language.

Figure 3 displays the fate and transport of contaminant from rectangular source on the $x z$-plane $(y=0)$ at $t=10$, $t=100$, and $t=500$ days under conditions by continuous injection, where the black line stands for $t=10$ days, the red line represents $t=100$ days, and the blue line denotes $t=500$ days. As expected, the pollution plume spreads out with time, and the plume has already reached the lower boundary of aquifers at $t=100$ days. The degree of spreading along $x$-axis is more than that along $z$-axis, and the migration speed of contaminant at the near field is lower than that of contaminant at the far field. The reason is due to the fact

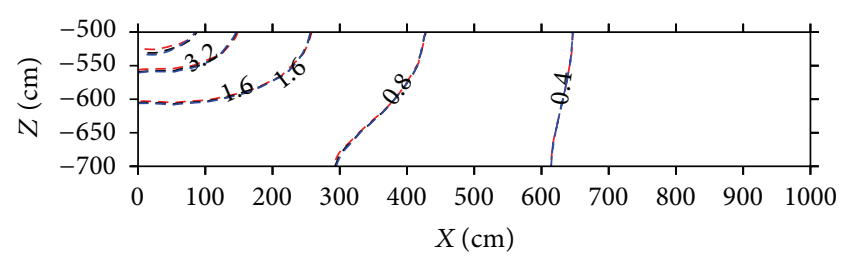

FIGURE 4: Contaminant concentration contours on the $x z$-plane $(y=0)$ under larger source area, unchanged source area, and smaller source area conditions at $t=100$ days for rectangular source geometry.

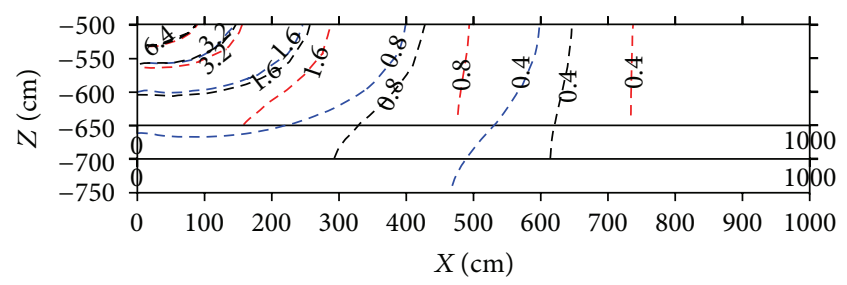

FIgURE 5: Contaminant concentration contours on the $x z$-plane $(y=0)$ under $H=150 \mathrm{~cm}, H=200 \mathrm{~cm}$, and $H=250 \mathrm{~cm}$ conditions at $t=100$ days for rectangular source geometry.

that there exists one-dimensional steady-state ground water flow along the $x$-axis, and the dispersion effect along $x$-axis $\left(D_{x}=2000\right)$ is larger than that along $z$-axis $\left(D_{z}=500\right)$. At initial time, due to the higher concentration gradient and gravity, the plume spread quickly both along $x$-axis and $z$ axis; however, this phenomenon is insignificant over time by continuous injection. These results agree with the findings by Cianci et al. [17] investigating contaminant transport through a saturated porous medium in a semi-infinite domain. In comparison with the work by Leij et al. [18], which illustrate that the relatively high maximum concentration occurs at the surface. This conclusion is obtained based on the surface condition transporting from a rectangular source, which is also indicated in this research.

Figure 4 illustrates contaminant concentration contours on the $x z$-plane $(y=0)$ under larger source area (red line), unchanged source area (black line), and smaller source area conditions (blue line) at $t=100$ days. The result of larger area is obtained under condition that rectangular source area is double, while the continuous injection strength reduces to half compared to the original source area. Similarly, smaller area means that rectangular source area reduces to half, while the continuous injection strength is double. From the figure, we can draw a conclusion that the influence of source area size decreases with the increase of distance to field; when the pollution plume migrates to a certain distance, the variation of source area size has no effect on plume migration. In order to make this problem clear, we conduct the following study.

Figure 5 presents contaminant concentration contours on the $x z$-plane $(y=0)$ under $H=150 \mathrm{~cm}$ (red line), $H=$ $200 \mathrm{~cm}$ (black line), and $H=250 \mathrm{~cm}$ (blue line) conditions at $t=100$ days, which shows the influence of aquifer thickness on pollutant migration. The pollution plume migrates slower with aquifer thickness increasing. In order to analyze 


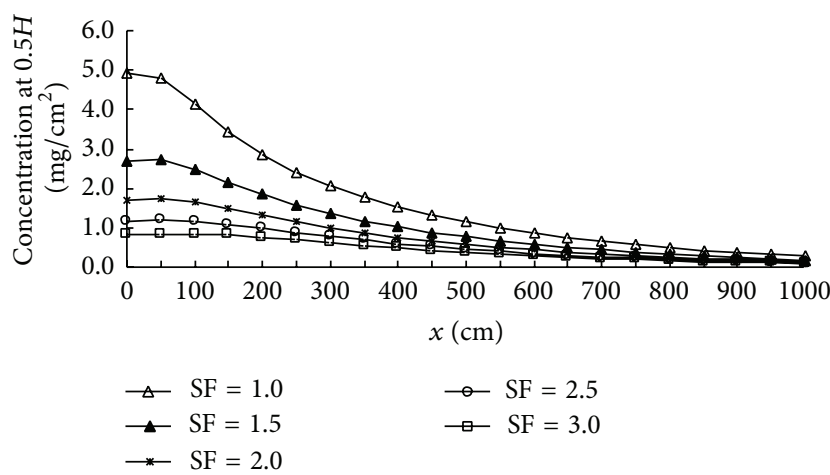

(a)

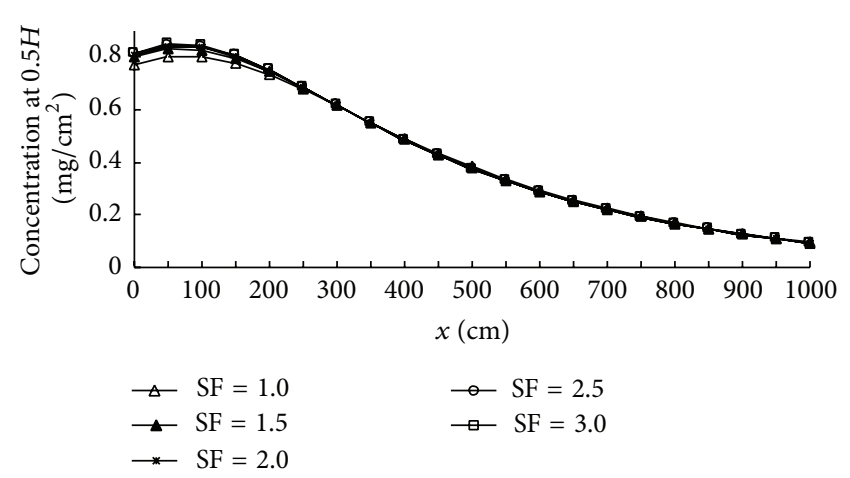

(b)

FIGURE 6: Contaminant concentration at half aquifer thickness under various scale factors at $t=100$ days for rectangular source geometry.

TABLE 2: The related calculation parameters for Figure 6.

(a)

\begin{tabular}{lcccc}
\hline & $x_{0}(\mathrm{~cm})$ & $y_{0}(\mathrm{~cm})$ & $I_{a}\left(\mathrm{mg} / \mathrm{cm}^{2}\right)$ & $H(\mathrm{~cm})$ \\
\hline $\mathrm{SF}=1.0$ & 50 & 25 & 100 & 100 \\
$\mathrm{SF}=1.5$ & 50 & 25 & 100 & 150 \\
$\mathrm{SF}=2.0$ & 50 & 25 & 100 & 200 \\
$\mathrm{SF}=2.5$ & 50 & 25 & 100 & 250 \\
$\mathrm{SF}=3.0$ & 50 & 25 & 100 & 300 \\
\hline
\end{tabular}

(b)

\begin{tabular}{lcccc}
\hline & $x_{0}(\mathrm{~cm})$ & $y_{0}(\mathrm{~cm})$ & $I_{a}\left(\mathrm{mg} / \mathrm{cm}^{2}\right)$ & $H(\mathrm{~cm})$ \\
\hline $\mathrm{SF}=1.0$ & 150 & 25 & $100 / 3$ & 300 \\
$\mathrm{SF}=1.5$ & 100 & 25 & 50 & 300 \\
$\mathrm{SF}=2.0$ & 75 & 25 & $200 / 3$ & 300 \\
$\mathrm{SF}=2.5$ & 60 & 25 & $250 / 3$ & 300 \\
$\mathrm{SF}=3.0$ & 50 & 25 & 100 & 300 \\
\hline
\end{tabular}

the comprehensive influence of both source area and aquifer thickness, we define a scale factor as follows:

$$
\mathrm{SF}=\frac{H}{L_{S}},
$$

where SF denotes scale factor; $H$ is aquifer thickness; $L_{S}$ stands for width of rectangular source (or major axis of elliptic source).

Figure 6 shows contaminant concentration at half aquifer thickness under various scale factors at $t=100$ days. The calculation parameters are listed in Table 2. The results in Figure 6(a) are obtained with a fixed rectangular source area under various aquifer thicknesses conditions (as shown in Table 2(a)). From the vertical perspective, the contaminant concentration at half aquifer thickness is decreasing with scale factor rising; besides differences of contaminant concentration decrease among various scale factors. From the horizontal perspective, differences of contaminant concentration among various scale factors become smaller with the distance to field increasing. Figure 6(b) is achieved with a fixed aquifer thickness under various rectangular sources

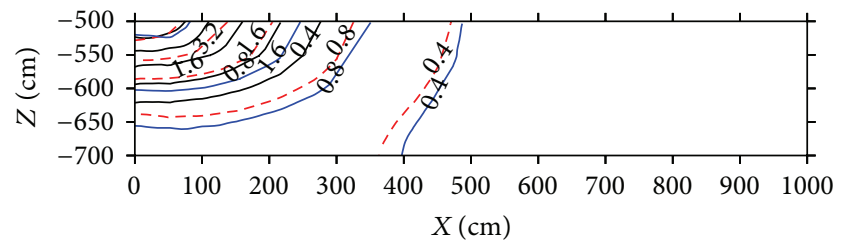

Figure 7: Contaminant concentration contours on the $x z$-plane $(y=0)$ under $t=10, t=100$, and $t=500$ days under conditions for elliptic source geometry.

conditions (as shown in Table 2(b)). The contaminant concentration at half aquifer thickness is almost unchangeable with scale factor increasing when the distance to field is greater than $250 \mathrm{~cm}$. In other words, the influence of source area size can be neglected when the distance to field is greater than $250 \mathrm{~cm}$.

3.2. Elliptic Source Type. Table 1 lists the related parameters for model simulation of elliptic source. Figure 7 illustrates contaminant concentration contours on the $x z$-plane $(y=0)$ under $t=10$ days (black line), $t=100$ days (red line), and $t=500$ days (blue line) conditions by continuous injection. The pollution plume spreads similarly to that of rectangular source geometry, but the speed of contaminant migration is slower than that of rectangular source type in both transverse and longitudinal directions. These results agree with the findings by Sim and Chrysikopoulos [12] based on a continuous source loading from elliptic source geometry in saturated, homogeneous porous media.

Figure 8 displays contaminant concentration contours on the $x z$-plane $(y=0)$ under various elliptic source areas at $t=100$ days (larger source area (red line), unchanged source area (black line), and smaller source area conditions (blue line)). The influence of source area size on pollution plume migration is insignificant. It is consistent with that of rectangular source type.

Figure 9 illustrates contaminant concentration contours on the $x z$-plane $(y=0)$ under $H=150 \mathrm{~cm}$ (red line), $H=$ $200 \mathrm{~cm}$ (black line), and $H=250 \mathrm{~cm}$ (blue line) conditions 


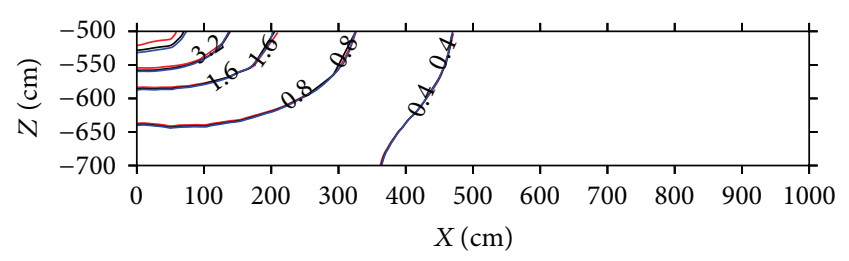

FIGURE 8: Contaminant concentration contours on the $x z$-plane $(y=0)$ under larger source area, unchanged source area, and smaller source area conditions at $t=100$ days for elliptic source geometry.

at $t=100$ days. The influence of aquifer thickness for elliptic source is the same as that of rectangular source; the pollution plume migrates fast with aquifer thickness decreasing. The influence of scale factor on pollution plume migration from elliptic source geometry is the same as that of scale factor from rectangular source geometry under the two parameter conditions listed in Table 2.

3.3. Comparative Analysis. Figure 10 displays the variation of $\mathrm{CR} / \mathrm{CE}$, defined as dimensionless coefficient indicating contaminant concentration of rectangular source type divided by contaminant concentration of elliptic source type, under various scale factors along transverse direction. The $\mathrm{CR} / \mathrm{CE}$ in the near field is smaller than that in the far field, and especially for $x=100 \mathrm{~cm}$, the value is almost equal to 1 . In other words, the contaminant concentration from rectangular source is nearly the same as that from elliptic source at $x=100 \mathrm{~cm}$ on water table, and the CR/CE is slightly decreasing with scale factor increasing. The research studied by Chrysikopoulos [19] also indicated that predictions of contaminant concentrations were sensitive to the source geometry for short downstream distance. However, when scale factor increases to a certain degree (i.e., $\mathrm{SF}=7.0$ ), the CR/CE keeps constant. In Figure 10(b), the situation is slightly different that the CR/CE increases with scale factor rising. However, the $\mathrm{CR} / \mathrm{CE}$ keeps constant when scale factor increased to $\mathrm{SF}=9.0$. The main reason is attributed to the fact that the same and continuous injection patterns make the differences of contaminant concentration between two source geometries in the near field insignificant on water table. Since contaminant migration from rectangular source geometry is slightly faster than that from elliptic source geometry as previously studied, so the difference between the two source geometries in the far field is significant, while it keeps constant if scale factor reaches a certain value. Assuming that if the lower boundary is infinite (i.e., the scale factor is big enough), both source geometries could be regarded as point source geometry. In this context, there would be no difference on contaminant migration between the two source geometries.

From the above discussion, a conclusion is obtained that the $\mathrm{CR} / \mathrm{CE}$ keeps constant under condition that the scale factor is equal to or greater than 9. Meaning that the rectangular source geometry can substitute for the elliptic source geometry under condition that scale factor is equal to

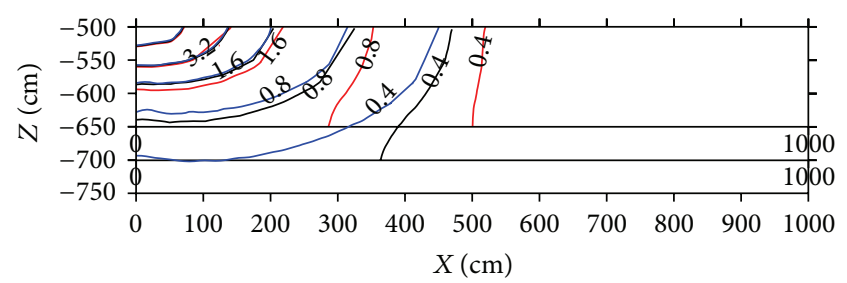

FIgURE 9: Contaminant concentration contours on the $x z$-plane $(y=0)$ under $H=150 \mathrm{~cm}, H=200 \mathrm{~cm}$, and $H=250 \mathrm{~cm}$ conditions at $t=100$ days for elliptic source geometry.

or greater than 9. In other words, we can use the data of contaminant migration from rectangular source geometry and the corresponding value of CR/CE to analyze and estimate the contaminant migration from elliptic source geometry.

\section{Summary}

Three-dimensional analytical solutions for contaminant transport in subsurface porous media from rectangular source geometry and elliptic source geometry were investigated, accounting for three-dimensional hydrodynamic advection-dispersion in a uniform flow field, first-order decay rates. The fate and transport of contaminant were simulated based on a continuous source loading. Several interesting solutions were obtained as follows.

(1) The migration of pollution plume from the two source geometries shows the same trend under the same aspect ratio, the equal source area size, and the uniform total pollution load condition, except that the migration of pollution plume from rectangular source geometry is faster than that from elliptic source geometry.

(2) The influence of source area size on pollution plume migration decreases with the distance to field increasing. In particular when pollution plume migrates to a certain distance, as far as five times of source side length $\left(L_{S}\right)$, the variation of source area size has no effect on pollution plume migration.

(3) The migration of pollution plume becomes slower with the increase of aquifer thickness for both source geometries. Compared with elliptic source geometry, the phenomenon of rectangular source geometry is more significant.

(4) The contaminant concentration is decreasing with scale factor rising, and the differences among various scale factors get smaller with the distance to field increasing.

(5) When scale factor is equal to or greater than 9, the $\mathrm{CR} / \mathrm{CE}$ would keep constant; meaning that we can use the data of contaminant migration from rectangular source geometry and the corresponding value of CR/CE to analyze and estimate the contaminant migration from elliptic source geometry. 


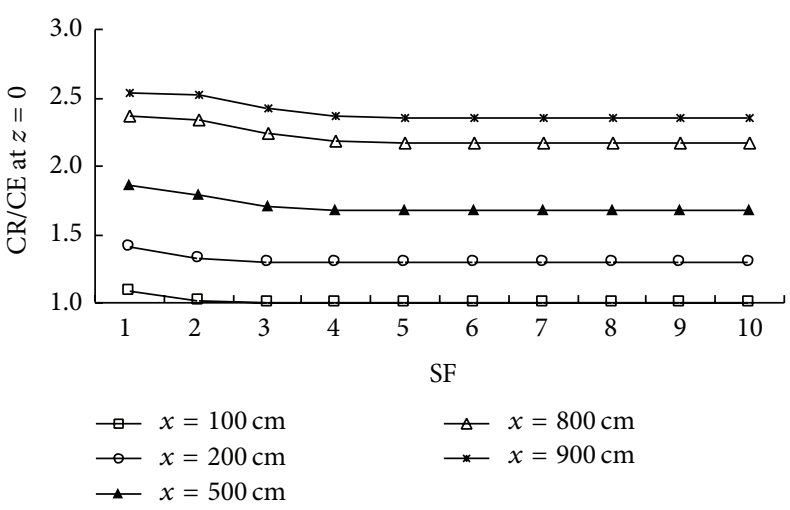

(a)

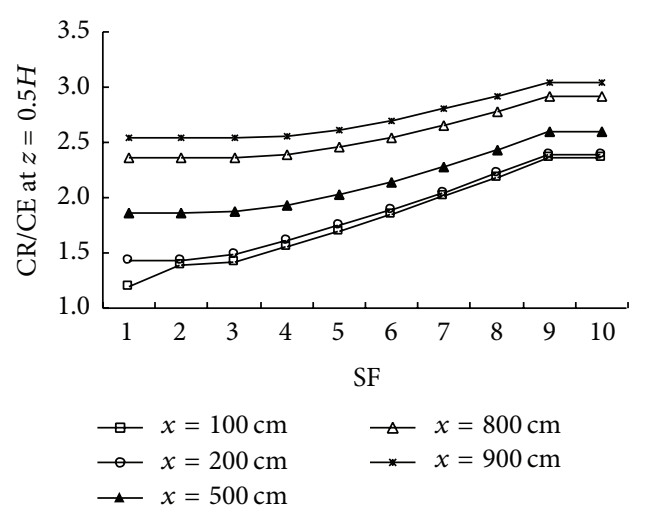

(b)

FIGURE 10: The variation of CR/CE with the increase of scale factor at various locations along transverse direction.

Since calculations of three-dimensional analytical solutions for contaminant transport from rectangular source geometry are simpler than those from elliptic source geometry (i.e., the rectangular source geometry requires little information on contaminated site and little calculation), we can use the data of contaminant migration from rectangular source geometry to obtain the contaminant transport from elliptic source geometry to simplify the actual engineering problem. The results would provide useful information and technical support for estimating the distribution of contaminant in the initial period of contaminated site investigation.

\section{Conflict of Interests}

The authors declare that there is no conflict of interests regarding the publication of this paper.

\section{Acknowledgments}

This work was supported by the National Natural Science Foundation of China (no. 41272248) and National Science and Technology Support Program (no. 2011BAC12B02). The authors would like to extend special thanks to the editor and the anonymous reviewers for their constructive comments and suggestions in improving the quality of this paper.

\section{References}

[1] S. K. Yadav, A. Kumar, and N. Kumar, "Horizontal solute transport from a pulse type source along temporally and spatially dependent flow: analytical solution," Journal of Hydrology, vol. 412-413, pp. 193-199, 2012.

[2] J. Mieles and H. Zhan, "Analytical solutions of one-dimensional multispecies reactive transport in a permeable reactive barrieraquifer system," Journal of Contaminant Hydrology, vol. 134-135, pp. 54-68, 2012.

[3] A. Ogata and R. B. Banks, "A solution of differential equation of longitudinal dispersion in porous media," Geological Survey Professional Paper (United States), vol. 411, pp. A1-A7, 1961.

[4] J.-P. Sauty, "An analysis of hydrodispersive transfer in aquifers," Water Resources Research, vol. 16, no. 1, pp. 145-158, 1980.
[5] M. T. Van Genuchten, "Analytical solutions for chemical transport with simultaneous adsorption, zero-order production and first-order decay," Journal of Hydrology, vol. 49, no. 3-4, pp. $213-$ 233, 1981.

[6] V. Batu, "A generalized two-dimensional analytical solution for hydrodynamic dispersion in bounded media with the first-type boundary condition at the source," Water Resources Research, vol. 25, no. 6, pp. 1125-1132, 1989.

[7] V. Batu, "A generalized two-dimensional analytical solute transport model in bounded media for flux-type finite multiple sources," Water Resources Research, vol. 29, no. 8, pp. 2881-2892, 1993.

[8] B. Sagar, "Dispersion in three dimensions: approximate analytic solutions.", Journal of the Hydraulics Division, ASCE, vol. 108, no. 1, pp. 47-62, 1982.

[9] P. A. Domenico, "An analytical model for multidimensional transport of a decaying contaminant species," Journal of Hydrology, vol. 91, no. 1-2, pp. 49-58, 1987.

[10] F. J. Leij, T. H. Skaggs, and M. T. van Genuchten, "Analytical solutions for solute transport in three-dimensional semiinfinite porous media," Water Resources Research, vol. 27, no. 10, pp. 2719-2733, 1991.

[11] V. Batu, "A generalized three-dimensional analytical solute transport model for multiple rectangular first-type sources," Journal of Hydrology, vol. 174, no. 1-2, pp. 57-82, 1996.

[12] Y. Sim and C. V. Chrysikopoulos, "Analytical solutions for solute transport in saturated porous media with semi-infinite or finite thickness," Advances in Water Resources, vol. 22, no. 5, pp. 507519, 1999.

[13] E. Park and H. Zhan, "Analytical solutions of contaminant transport from finite one-, two-, and three-dimensional sources in a finite-thickness aquifer," Journal of Contaminant Hydrology, vol. 53, no. 1-2, pp. 41-61, 2001.

[14] J. Chen, Y. Liu, C. Liang, C. Liu, and C. Lin, "Exact analytical solutions for two-dimensional advection-dispersion equation in cylindrical coordinates subject to third-type inlet boundary condition," Advances in Water Resources, vol. 34, no. 3, pp. 365374, 2011

[15] J. Chen, J. Chen, C. Liu, C. Liang, and C. Lin, "Analytical solutions to two-dimensional advection-dispersion equation in cylindrical coordinates in finite domain subject to first- and third-type inlet boundary conditions," Journal of Hydrology, vol. 405, no. 3-4, pp. 522-531, 2011. 
[16] C. Zhao, B. E. Hobbs, and A. Ord, "Effects of domain shapes on the morphological evolution of nonaqueous-phase-liquid dissolution fronts in fluid-saturated porous media," Journal of Contaminant Hydrology, vol. 138-139, pp. 123-140, 2012.

[17] R. Cianci, M. Massabó, and O. Paladino, "An analytical solution of the advection dispersion equation in a bounded domain and its application to laboratory experiments," Journal of Applied Mathematics, vol. 2011, Article ID 493014, 14 pages, 2011.

[18] F. J. Leij, E. Priesack, and M. G. Schaap, "Solute transport modeled with Green's functions with application to persistent solute sources," Journal of Contaminant Hydrology, vol. 41, no. 1-2, pp. 155-173, 2000.

[19] C. V. Chrysikopoulos, "Three-dimensional analytical models of contaminant transport from nonaqueous phase liquid pool dissolution in saturated subsurface formations," Water Resources Research, vol. 31, no. 4, pp. 1137-1145, 1995. 


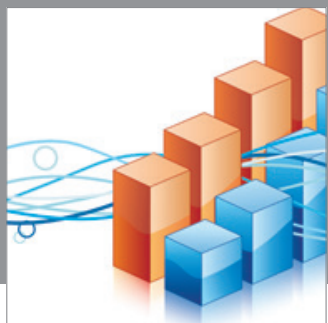

Advances in

Operations Research

mansans

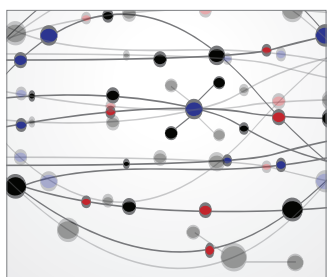

The Scientific World Journal
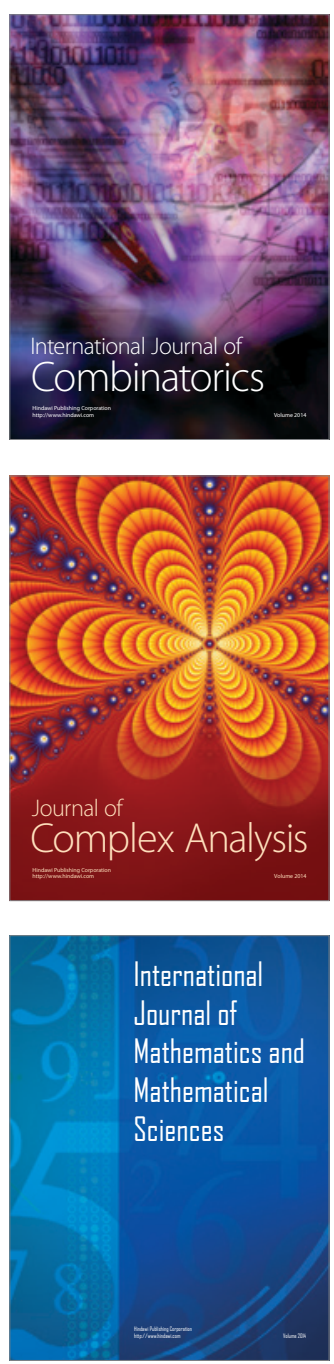
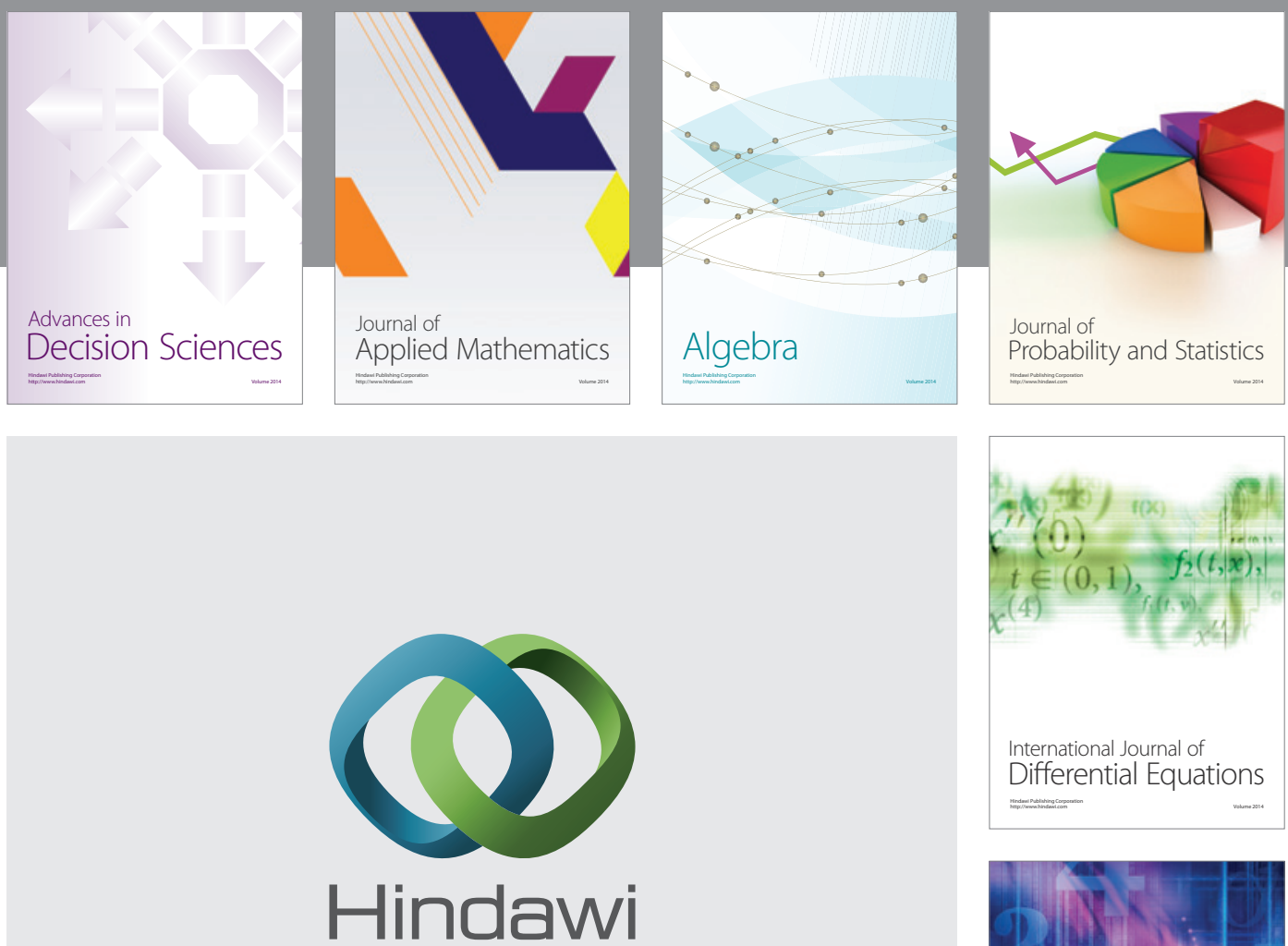

Submit your manuscripts at http://www.hindawi.com
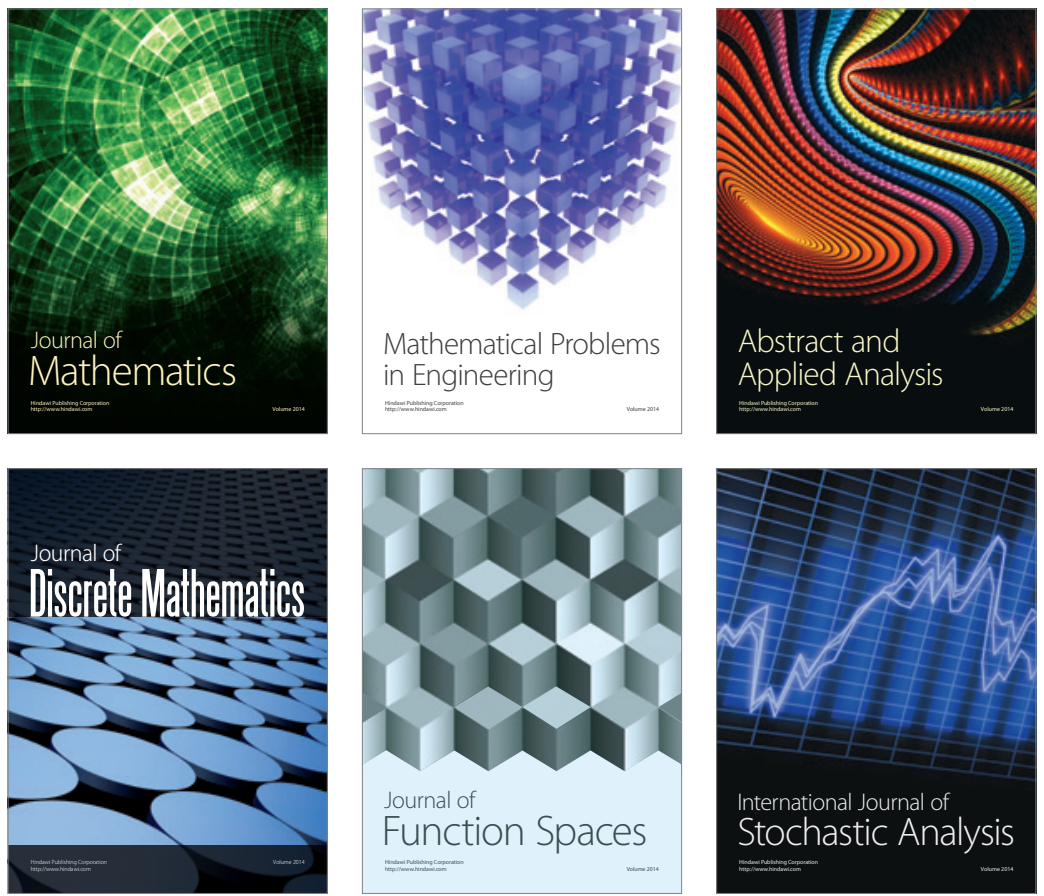

Journal of

Function Spaces

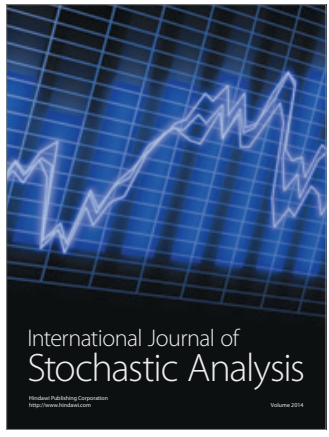

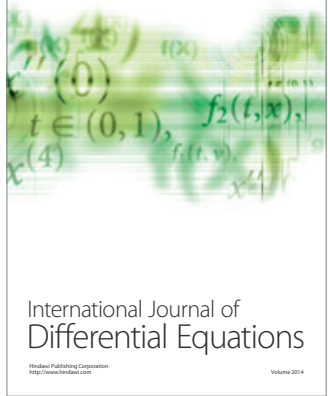
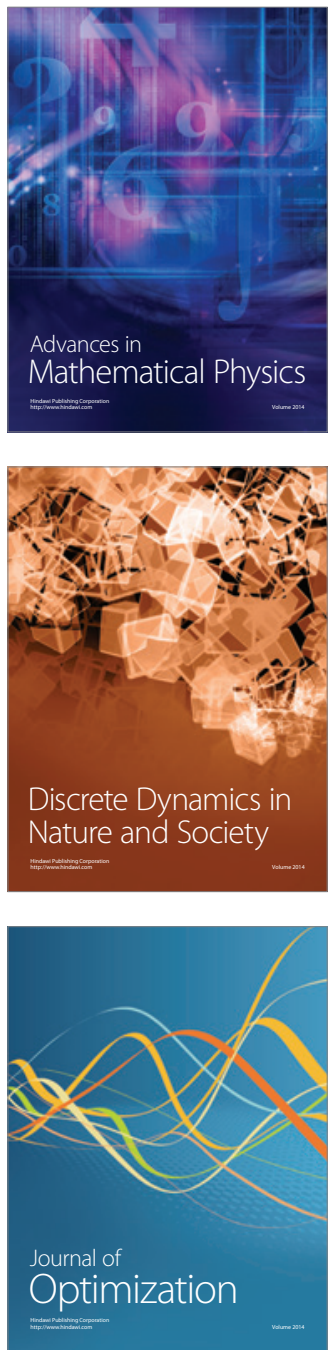